\title{
Cellular Response to Inflammation at the Limbus
}

\author{
GILBERT SMOLIN
}

San Francisco

\begin{abstract}
Summary
The role in ocular (limbal) inflammation of the rich vascular and lymphatic supply is discussed in detail. The distribution of lymphocytes, mast cells, Langerhans cells, macrophages and other cellular elements are discussed as well as their individual and collective response to inflammation whether due to infectious or non-infectious disease states. Among the non-infectious causes of limbal cellular inflammation are the four types of hypersensitivity response.
\end{abstract}

Inflammation results in the dilation of blood vessels, the leakage of fluid into the extravascular spaces, and the migration of leukocytes from the blood stream into these spaces. Specific chemical substances are frequently released during inflammation. These substances tend to heighten or perpetuate the response.

The vascular component of inflammation is the most important because the external eye (i.e. limbus) is so richly supplied with vessels, many of them grossly visible in tissues such as the conjunctiva.

The muscular branches of the ophthalmic artery with their anterior ciliary branches form the rich blood supply of the limbus. ${ }^{1}$ The arterial channels are composed of arterioles and end in a complex capillary network at the limbus. The conjunctival vessels form a superficial marginal plexus which branches and as it progresses toward the limbus forms the terminal peripheral corneal arcades and recurrent vessels which run posteriorly to supply 3-6 mm of the perilimbal area. Immune complexes deposit in the extravascular spaces around these vessels.

Ocular inflammation first dilates the blood vessels and then allows for the leakage of serum and blood elements, namely, C-reactive protein and immunoglobulins, macrophages, neutrophilic polymorphonuclear leukocytes, and lymphocytes, into the extravascular spaces.

Lymphatics are also present in the limbus. The bulbar conjunctival lymphatics begin at the limbus in a series of arcades. There are two interconnected plexuses (superficial and deep) that drain toward the palpebral commissures, where they join with the lymphatics of the lids. Antigenic material and lymphocytes pass through these channels, ultimately reaching the draining lymph nodes and/or central reticuloendothelial organs, where immunologic responses are initiated.

The conjunctiva has a rich supply of immunoglobulins A, G and M (IgA, IgG, and IgM), probably derived from the rich vascular supply and abundant plasma cells.

The vasculature may also be the source of the complement, properdin, and properdin factor that are present in the tear film..$^{2,3}$

The sequence of vascular events in inflammation consists of preliminary vasoconstriction, then arteriolar dilation, oedema formation, blood cell aggregation, sticking of blood cells to the vascular endothelium, and 
eventually, stasis. The minute vessels are not subject to neuronal regulation, but they are instead affected by chemical substances that are liberated from cellular stores or formed $d e$ novo (i.e. leukotrienes, histamine, and prostaglandins (PG)). The larger vessels are subject to neurogenic mechanisms of vasomotor activity.

Most inflammatory reactions are biphasic, that is, there is an immediate response that reaches its maximum eight to ten minutes after the application of a noxious stimulus. This is followed by a levelling-off period of variable duration, during which homeostasis can be achieved. This, in turn, is followed by a late phase of vessel dilation and increased vascular permeability that unfolds over a period of hours and culminates in the infiltration of the tissue with blood elements (i.e. leukocytes, C-reactive protein, complement).

The first phase is mediated by histamine or histamine-like substances and the second phase by the kinins and prostaglandins.

The submucosal tissue of the conjunctiva contains mast cells. In response to an allergen, injury, or other appropriate stimuli, these cells release histamine, as well as a variety of other substances that can cause blood vessel dilation and increased vascular permeability noted above by interacting with the $\mathrm{H}_{1}$ receptors present on the ocular surface. Stimulation of the $\mathrm{H}_{1}$ receptor may also result in pruritis, PG generation, and an increase in cyclic guanosine monophosphate (GMP). The latter causes enhanced release of the pharmacologic mediators from the mast cells or circulating basophils. $\mathrm{H}_{2}$ receptor stimulation increases mucus secretion (a feature common to atopic ocular disease) and cyclic adenosine monophosphate (AMP) production, which in turn decreases the release of pharmacologic mediators from the mast cells and basophils. Stimulation of either $\mathrm{H}$ receptor can result in vasodilation.

The activation and degranulation of mast cells require $\mathrm{Ca}+$. The serine esterase in the cell wall is eventually broken down to lysophosphatidyl choline and arachidonic acid. The arachidonic acid is subsequently broken down by the cyclooxygenase pathway to PF and thromboxanes or by the lipoxy- genase pathway to hydroxyeicosatetraenoic acid (HETE) or leukotrienes.

The mediators released by the mast cell may be preformed, secondarily formed (generated by the interaction of primary mediators and nearby cells and tissues), or granule matrix derived (which are preformed but do not readily dissociate from the granule after discharge). The preformed mediators are released into the tissue immediately. These include histamine, eosinophil chemotactic factors of anaphylaxis, neutrophil chemotactic factor, superoxide anions, and kininogenase. The kininogenase results in the formation of kinins, which can alter vascular permeability dramatically.

Several of the secondary mediators are formed immediately (PG) or within minutes (leukotrienes) and, unlike histamine, may last for hours. The secondary mediators have effects that enhance inflammation by

(1) increasing cyclic GMP (PF G $\mathrm{P}_{2}$ and $\mathrm{F}_{2 \mathrm{a}}$ ),

(2) causing chemotaxis of eosinophils and neutrophils (HETE, PG $1_{2}$, and leukotriene $\mathrm{B}_{4}$ ),

(3) causing platelet aggregation (thromboxane $\mathrm{A}_{2}$ ),

(4) increasing mucus secretion $\left(\mathrm{PGF}_{2 \mathrm{a}}, \mathrm{E}_{1}, \mathrm{I}_{2}\right.$, $D_{2}$ and $A_{2}$, HETE, and leukotrienes $C$ and D)

(5) augmenting mediator release $\left(\mathrm{PG} \mathrm{F}_{2 \mathrm{a}}\right.$ and HETE)

(6) increasing vascular permeability $\left(\mathrm{PG} \mathrm{E}_{2}\right.$ and leukotrienes $C, D$, and $E$ ), and

(7) causing vasodilation ( $P G \mathrm{E}_{2}$ and leukotrienes $\mathrm{C}, \mathrm{D}$, and $\mathrm{E}) .^{5}$

The inflammatory reaction is regulated by substances that neutralise the effect of the above mediators (i.e. peroxidases, arylsulfatases, and certain prostaglandins) and that elevate cyclic AMP, which in turn stabilises the mast cell membrane (certain prostaglandins, histamine). PGE may act as a negative feedback inhibitor of T-cell proliferation, lymphokine production, macrophage activity, and natural killer cell cytotoxicity. ${ }^{6}$

Of the various stimuli that can produce inflammation in the limbus those that activate complement probably have the most farreaching effects. This complex substance, 
present in the external ocular tissue, can become bound to antigen-antibody complexes, to simple aggregates of foreign proteins, and to certain toxic substances. The union of complement with these substances triggers enzymatic reactions that actually cause the formation of holes in cellular membranes and the release of chemotactants (attracting neutrophils) and anaphylatoxins. The integrity of the vascular endothelium may become permanently altered (which attract eosinophils, basophils, and neutrophils). The polymorphonuclear leukocytes attracted to this area can release a variety of proteolytic enzymes that cause tissue necrosis.

When damaged, the conjunctival epithelium can release a thymocyte-activating factor (ETAF), which can attract polymorphonuclear leukocytes, fibroblasts, and lymphocytes. This substance has interleukinl-like properties. ${ }^{8}$

Another interesting cell type found in the limbal epithelium are the Langerhans cells or dendritic cells ( $D$ cells). They play a major role in the processing of antigen presented via the epithelial surface and they carry unique histocompatibility antigens (Ia) that are of importance in stimulating $\mathrm{T}$ and $\mathrm{B}$ lymphocytes. ${ }^{9}$ These cells bind antigens and carry them by way of the lymphatics to the draining lymph nodes, which leads to host sensitisation. ${ }^{10}$ They also stimulate helper T cells and B cells to participate in humoral immune responses. ${ }^{11}$ The stimulated $\mathrm{T}$ and $\mathrm{B}$ lymphocytes in the regional lymph nodes then migrate via the blood stream to the ocular adnexa. The $\mathrm{T}$ cells tend to home to submucosal sites in the conjunctiva, whereas B cells home to the lacrimal gland and accessory lacrimal gland epithelia. ${ }^{12}$ The $\mathrm{B}$ cells can produce immunoglobulins at these sites, particularly $\operatorname{IgA} .^{13}$

In different diseases, different populations of $\mathrm{T}$ cells can be seen (i.e. helper cells, suppressor cells) in the conjunctival submucosal tissue. ${ }^{14}$

Foreign substances can be processed locally by the musocal immune defence system. The tissue associated with this system is called the mucosal-associated lymphoid tissue (MALT). In the adnexa, the conjunctival-associated lymphoid system (CALT) is in histologically distinct areas. The epithelium over these CALT sites shows elongated microvilli with few microplicae. The underlying lymphoid nodules are multiple and packed with lymphocytes. Antigens are preferentially processed at these sites.

In addition to the active role of the components noted above in causing ocular inflammation, the limbal tissue may be an innocent bystander for the deposition of immune complexes. The anatomy of the vasculature at the corneal limbus may account for the deposition of these complexes, which in turn results in the binding of complement, the attraction of polymorphonuclear leukocytes, and subsequent inflammation.

The cellular response of the limbus can vary quantitatively and qualitatively depending upon the stimulus. Infectious and noninfectious processes may influence this response. In general, nongranulomatous bacterial, fungal and parasitic infections attract neutrophils; granulomatous bacterial infections attract epithelioid cells, lymphocytes, macrophages and possibly giant cells; and viral infections a mononuclear response (unless necrosis is extensive enough to attract neutrophils). Noninfectious, nonimmune aetiologies of limbal disease include toxic and degenerative processes. Immunologic responses at the limbus can be divided in the four classical hypersensitivity types. In the type 1-anaphylactic response the commonest ocular manifestations occur with the hay fever type of anaphylactic reaction. There is rapid vascular congestion and conjunctival chemosis. The conjunctiva looks milky or pale pink. All these signs are the sequelae of the release of the vasoactive amines.

In the vernal or atopic type of keratoconjunctivitis a mononuclear cell infiltrate can occur at the limbus. The presence of lymphocytes and macrophages suggest that this type of reaction has a slightly different pathogenesis.

A papillary limbal response in vernal or atopy is the result of marked hyperplasia of connective tissue and of inflammatory infiltration (with eosinophils, mast cells, basophils, lymphocytes, and macrophages predomination).

Limbal nodules may be single or multiple. 
They appear as small, semitransparent, smooth, gelatinous elevations, usually in the interpalpebral fissure. Their corneal edge is sharp but their conjunctival edge blends gradually with the normal tissue. Their colour may vary from grayish white to pink depending upon the degree of vascularisation. These limbal masses may continue to grow and coalesce until they completely surround the cornea. Occasionally they extend centrally-with from minimal to extensive corneal invasion. ${ }^{17}$

Thickening, broadening, and opacification at the upper limbus may extend onto the cornea as a semitransparent hood. Peripheral vascularisation of this area may extend into the cornea as a micropannus, rarely as a gross pannus. The pannus eventually clears, leaving ghost vessels and a few gray opacities. Occasionally the gelatinous limbal nodules extend centrally to cover the entire cornea, and in a few cases the corneal nodules have not been connected to the limbus.

The limbal white points (Horner's points or Trantas' dots) present in vernal or atopy are small, grayish white to whitish yellow dots appearing singly or in large numbers, usually at the upper limbus. They are sometimes seen on the bulbar conjunctiva and semilunar folds, however, and very rarely on the tarsal conjunctiva. They are present in about 69 per cent of the cases of mixed vernal keratoconjunctivitis (VKC) in 41 per cent of limbal VKC, and in 21 per cent of palpebral VKC. They usually last for as long as a week. Pathologically they are small cysts filled with eosinophils, granules, and epithelial cells undergoing rapid degeneration. They are the tip of the iceberg whose bulk lies in the deeper layers of the epithelium.

In the limbal form of $\mathrm{VKC}$, cysts and minute marginal 'pits' also occur. The cysts are ovoid, closely packed in some areas, contain clear, colourless fluid, and appear to be elevated. They are true cysts formed by the apposition of two epithelial layers between papillae and they contain mucin as well as epithelial and inflammatory cells. The marginal 'pits' appear as transparent, round to oval, glassy spots in the opaque limbus. They are not depressed and represent areas in which the limbal infiltration has almost returned to normal. They are not to be confused with the
Herbert's peripheral pits of trachoma, which are larger and permanent.

Type II-Cytotoxic Response is the one that participates in the immune protection of the host from pyogenic bacteria, transplanted tissue, and tumour cells. The action of the antigen on the foreign cell, combined with antibody (IgG or IgM), causes the death of the affected cell by bringing about its contact with phagocytes or killer cells. Phagocytosis is accomplished by a reduction in surface charge; opsonic adherence directly through a portion of the antibody, the phagocytes adhering to antibody without the assistance of complement; or immune adherence through bound $\mathrm{C} 3 \mathrm{~b}$, the phagocytes adhering to antibody with the assistance of complement. The activation of complement occasionally occurs and results in cell death.

Some examples of these cytotoxic reactions are organ transplantation reaction (in part), many drug reactions, Mooren's ulcer, and mucous membrane pemphigoid.

In Mooren's ulceration plasma cells are abundant at the limbus. ${ }^{18}$

In cicatricial pemphigoid, as well as in bullous pemphigoid of the skin, the basement membrane of the epithelium is under attack by autoimmune processes. By the use of immunofluorescent techniques, complement, IgG, IgM, and IgA have all been found to be localised in the basement membrane zone. Neutrophils are attracted to these sites.

In the Type III-Immune Complex Response the injured cells or tissue are innocent bystanders and do not possess the antigenic determinants that can combine with the antibody that caused the injury. The antibody reacts with its antigen, and the resulting immune complex binds complement, attracts PMNs and then directly or indirectly injures tissues that are in the vicinity. The result can be catarrhal ulceration; or peripheral corneal infiltrates in a variety of conditions, e.g. rheumatoid arthritis, polyarteritis nodosa. The distribution of the injury to the tissue is determined by the sites where the immune complex form or are deposited (i.e. limbus).

The mechanism of immune complexinduced injury is complicated. The size of the complex is important: Large complexes are easily phagocytised by the reticuloendothelial 
system and do not localise in tissues; small complexes persist in the circulation but may be too small to localise in tissues. It is the intermediate sizes that localise in tissues, but the vascular permeability must increase before they can localise in the extravascular areas, e.g. the limbus. The vasoactive amines may play a crucial role in increasing this permeability, and platelets, complement, anaphylatoxins, and mast cell or basophils combined with IgE may also be sources of these vasoactive amines.

Previous damage (nonimmunologic or immunologic) to the blood vessels can also increase their permeability and pave the way for the subsequent deposition of immune complexes in the surrounding tissues (the Auer reaction).

The Type IV-Cell-Mediated Immune (CMI) response is due entirely to the activity of thymus-dependent lymphocytes.

The lymphocyte responds primarily to fixed rather than soluble antigens, participating in reactions to many bacteria, viruses, fungi, tissue allografts, and neoplasms. There are two types of this delayed hypersensitivity response. The two types are the classic, tuberculin type in which $T$ cells and macrophages participate; and the cutaneous, basophilic type previously called the Jones-Mote reaction.

The CMI response plays a role in protecting against infections and tumours and in destroying transplanted tissue.

\section{References}

1 Wolff E: 'Anatomy of the Eye and Orbit'. Philadelphia: Saunders WB, 1961, pp. 379-381.

${ }^{2}$ Bluestone R: Lacrimal immunoglobulins and complement quantified by counter-immuno- electrophoresis. Br J Ophthalmol 1975, 59: 279_ 83.

${ }^{3}$ Smolin G and O'Connor GR: 'Ocular Immunology', Boston, Little, Brown and Co, 1987, p. 76.

${ }^{4}$ Abelson $\mathrm{MB}$ and Udell $\mathrm{IJ}: \mathrm{H}_{2}$-receptors in the human ocular surface. Arch Ophthalmol 1981, 99: 302-5.

${ }^{5}$ Marom Z and Casale TB: Mast cells and their mediators. Ann Allergy 1983, 50: 367-70.

${ }^{6}$ Goodwin JS and Ceuppers J: Regulation of the immune response by prostaglandins. J Clin Immunol 1983, 3: 295-9.

${ }^{7}$ Gamble CN, Aronson SB, Brescia FB: Experimental uveitis. Arch Ophthalmol 1970, 84: 32130.

${ }^{8}$ Grabner G, Luger TA, Smolin G, Openheim JJ: Corneal epithelial cell-derived thymocyte activity factor. Invest Ophthalmol Vis Sci 1982, 23: 75763.

${ }^{9}$ Gillette TE, Chandler JW, Greiner JV: Langerhans of the ocular surface. Ophthalmology 1982, 89: 700-10.

${ }^{10}$ Chandler JW and Gillette TE: Immunologic defense mechanisms of the ocular surface. Ophthalmology 1982, 89: 700-10.

11 Silberberg-Sinakin E, Baer RL, Thorbecke GJ: Langerhans cells: A review of their nature with emphasis on their immunologic functions. Prog Allergy 1978, 24: 268-94.

${ }^{12}$ Chodirker WB and Thomasi TB Jr: Gamma globulins: Quantitative relationships in human serum and nonvascular fluids. Science 1963, 142: 1080-1.

13 Jackson EE, Lally ET, Nakamura MC, Montgomery PC: Migration of IgA bearing lymphocytes into salivary glands. Cell Immunol 1981, 63: 203-9.

${ }^{14}$ Bhan AK, Fujikawa L and Foster CS: T-cell subsets and Langerhans cells in normal and diseased conjunctiva. Am J Ophthalmol 1982, 94: 205-12.

${ }^{15}$ Chandler JW and Axelrod AJ: Conjuncitiva-associated lymphoid tissue. In O'Connor GR (ed.), 'Immunologic Disease of the Mucous Membranes'. New York: Masson, 1980, pp. 63-70.

${ }^{16}$ Bienenstock J, Johnson N, Perey DTE: Bronchial lymphoid tissue 1. Morphologic characteristics. Lab Invest 1973, 28: 686-92.

${ }^{17}$ Hogan MJ: Atopic keratoconjunctivitis. Am J Ophthalmol 1953, 36: 937.

${ }^{18}$ Brown SL: Mooren's ulcer. Br J Ophthalmol 1975, 59: 670. 